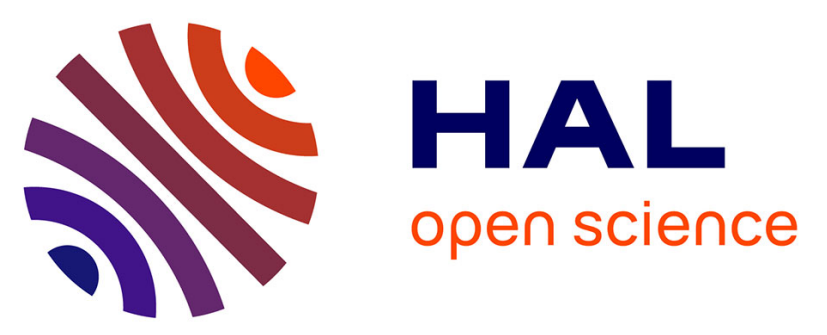

\title{
Morphology and Growth Mechanisms of Self-Assembled Films on Insulating Substrates: Role of Molecular Flexibility and Entropy
}

\author{
Julian Gaberle, David Gao, Alexander L Shluger, Ania Amrous, Franck \\ Bocquet, Laurent Nony, Franck Para, Christian Loppacher, Simon Lamare, \\ Frederic Cherioux
}

\section{To cite this version:}

Julian Gaberle, David Gao, Alexander L Shluger, Ania Amrous, Franck Bocquet, et al.. Morphology and Growth Mechanisms of Self-Assembled Films on Insulating Substrates: Role of Molecular Flexibility and Entropy. Journal of Physical Chemistry C, 2017, 121 (8), pp.4393-4403. 10.1021/acs.jpcc.6b12738 . hal-01702376

\section{HAL Id: hal-01702376 https://hal.science/hal-01702376}

Submitted on 7 Feb 2018

HAL is a multi-disciplinary open access archive for the deposit and dissemination of scientific research documents, whether they are published or not. The documents may come from teaching and research institutions in France or abroad, or from public or private research centers.
L'archive ouverte pluridisciplinaire HAL, est destinée au dépôt et à la diffusion de documents scientifiques de niveau recherche, publiés ou non, émanant des établissements d'enseignement et de recherche français ou étrangers, des laboratoires publics ou privés. 


\title{
Morphology and Growth Mechanisms of Self-Assembled Films on Insulating Substrates: Role of Molecular Flexibility and Entropy
}

\author{
Julian Gaberle, ${ }^{* \dagger \odot}$ David Z. Gao, ${ }^{*, \dagger}$ Alexander L. Shluger, ${ }^{\dagger,+}$ Ania Amrous, ${ }^{\S}$ Franck Bocquet, ${ }^{\S}$ \\ Laurent Nony, ${ }^{\S}$ Franck Para, ${ }^{\S}$ Christian Loppacher, ${ }^{*}$, Simon Lamare,, and Fréderic Cherioux ${ }^{\|}$ \\ ${ }^{\dagger}$ Department of Physics and Astronomy, University College London, Gower Street, London WC1E 6BT, United Kingdom \\ ${ }^{*}$ WPI-AIMR, Tohoku University, 2-1-1 Katahira, Aoba-ku, Sendai 980-8577, Japan \\ ${ }^{\S}$ Aix-Marseille Université CNRS, IM2NP UMR 7334, 13397 Marseille, France \\ "Institut FEMTO-ST, Université de Bourgogne Franche-Comté, CNRS, 15B Avenue des Montbouçons, Besancon F-25030 CEDEX, \\ France
}

\section{Supporting Information}

ABSTRACT: We studied the effect of molecular flexibility on the morphology and growth mechanisms of self-assembled films on an insulating substrate using a combination of experimental and theoretical methods. 1,3,5-Tri-(4cyano-4,4 biphenyl)-benzene (TCB) and 1,4-bis(cyanophenyl)-2,5-bis(decyloxy) benzene ( $\mathrm{CDB})$ molecules were deposited on a $\mathrm{KCl}(001)$ surface and imaged using noncontact atomic force microscopy (NC-AFM). Both molecules were designed to contain the same anchoring groups and benzene ring structures, yet CDB self-assembled structures were observed to grow from step edges, while TCB self-assembled structures grew as islands on the clean terrace and from step edges. Density functional theory (DFT) and atomistic molecular dynamics simulations were performed to understand this qualitative difference in growth modes. Calculations of free energies of dimer formation and step adhesion provide an insight into the role played by entropy loss in the morphology and growth modes of self-assembled films.

\section{INTRODUCTION}

Understanding adsorption and self-assembly of organic molecules on insulating surfaces is important for many applications, including sensor technologies, ${ }^{1,2}$ coatings, ${ }^{3}$ catalysis, ${ }^{4}$ and molecular electronics. ${ }^{5-7}$ In order to target these applications, the morphology and growth mechanisms of self-assembled films need to be controlled. While a high level of control over molecular structures has been achieved on metal surfaces $^{8-14}$ and semiconductors, ${ }^{15}$ many applications involve bulk insulators.

Previous experimental and theoretical studies on insulating surfaces have demonstrated that the formation of selfassembled film structures requires a well-controlled balance between molecule-molecule and molecule-surface interactions. ${ }^{16-22}$ Furthermore, the relative strengths of these interactions have been shown to have a direct effect on growth mechanisms. ${ }^{23}$ Recent studies emphasize the role of polar functional groups and their interaction with surface ions ${ }^{24}$ and entropic contributions to adsorption free energy. ${ }^{25}$ Thus, both how a molecule interacts with a surface ${ }^{17}$ and its conformational flexibility and ensuing entropic effects may play an important role in the design of molecules capable of selfassembly. In this paper we address both of these effects using experimental observations with noncontact atomic force microscopy (NC-AFM) and theoretical simulations.
The current status of NC-AFM studies of structures and selfassembly of organic molecules at ionic surfaces has been recently reviewed in ref 26 . Most of these studies are performed at room temperature where molecules are mobile and can selfassemble. However, the speed of self-assembly prevents observation of the intermediate stages of this complex process with molecular resolution as NC-AFM is too slow for this purpose. This gap can be partially filled by computer simulations of adsorption and self-assembly of molecules. However, these studies also suffer from a time gap between the nanoseconds, which can be afforded by molecular dynamics (MD) simulations and much longer times required for nucleation and growth of molecular surface structures. This gap can be closed using, e.g., Kinetic Monte Carlo, ${ }^{27,28}$ but this approach is feasible so far only for small or coarse-grained molecules and relatively simple processes. ${ }^{29}$ For larger molecules (about 100 atoms) most of the effort has been focused on developing methods for accurate treatment of the van der Waals interaction between molecules and surfaces, ${ }^{30,31}$ which improved the accuracy of prediction of adsorption enthalpies. Recent theoretical studies of the adsorption of individual 1,4-bis(cyanophenyl)-2,5-bis(decyloxy)benzene

Received: December 19, 2016

Revised: February 8, 2017

Published: February 9, 2017 
(CDB) molecules on the $\mathrm{KCl}$ (001) surface have shown that molecular flexibility affects significantly the entropy loss during adsorption and the adsorption free energy. ${ }^{25}$ The importance of entropy changes when considering the free energy of adsorption has been long recognized, ${ }^{32}$ and entropy changes have been measured experimentally for small molecules on oxide surfaces. ${ }^{33}$ Entropic effects have even been shown to determine the final structure formed by adsorbed molecules. ${ }^{34}$ It has been proposed that there is an intrinsic relationship between the flexibility and self-assembly of peptide surfactants. ${ }^{35}$ However, calculations of free energy for adsorption of organic molecules at insulating surfaces are still rare and hampered by the lack of force fields and computational expense of such calculations. Finding the right balance between elucidating general issues of self-assembly on specific systems and computational cost of such studies is difficult. The specific example used in this work highlights some general features important for our understanding of the mechanisms of selfassembly of rigid and flexible molecules functionalized by polar groups and suggests efficient computational strategies for elucidating structures of self-assembled monolayers.

This study is motivated by the experimental investigations of adsorption and self-assembly of 1,3,5-tri-(4-cyano-4,4-biphenyl) benzene (TCB) and 1,4-bis(cyanophenyl)-2,5-bis(decyloxy) benzene ( $\mathrm{CDB}$ ) molecules on the $\mathrm{KCl}(001)$ surface. The more rigid TCB molecule belongs to a special class of triangular "tripod" molecules, which were previously observed to form various regular $2 \mathrm{D}$ polymorphs. ${ }^{36-41}$ It contains the same functional groups as the previously studied flexible CDB molecule ${ }^{22}$ but does not possess flexible hydrocarbon chains. Both molecules were deposited onto $\mathrm{KCl} \mathrm{(001)} \mathrm{at} \mathrm{room}$ temperature and imaged using NC-AFM. At low coverage, CDB molecules were observed to fully decorate step edges, while TCB molecules produced hazy, partially covered step edges and two distinct types of periodic monolayer structures.

In order to understand the film structures and get some insight into its growth mechanisms and dynamics, we used the following strategy. As the dynamics of a system of this scale can only be studied using force fields, we derived such force fields for the molecule-surface interaction in refs 25 and 42 . Because the balance of interactions is crucial for our predictions, we first need to test our force fields in action. Using the information provided by experiment, we first modeled the molecular diffusion, the interaction of $\mathrm{CDB}$ and TCB molecules with the surface steps, and the pairwise interaction between the TCB molecules. Qualitative agreement with experiment gives us confidence in the force fields and insight into the importance of entropic effects in these processes. Then we use MD simulations of large molecular assemblies to investigate the main structural motifs formed by clusters of several TCB molecules. These motifs are used to construct prototype models of periodic molecular structures. The stability of these structures is tested using geometry optimization and MD simulations. The predicted structure is in good agreement with the experimental data confirming the validity of our approach. The results also highlight the importance of including entropic effects in modeling the adsorption and self-assembly of organic molecules at surfaces.

\section{METHODS}

Noncontact Atomic Force Microscopy. The synthesis of CDB and TCB molecules is described in detail in ref 22. Singlecrystal $\mathrm{KCl}$ (MaTecK GmbH, 52428 Jülich, Germany) was cleaved ex situ, quickly introduced into UHV, and annealed to $240{ }^{\circ} \mathrm{C}$ for $1 \mathrm{~h}$ in order to obtain atomically clean surfaces with large terraces. Molecules were deposited by evaporation from home-built crucibles at evaporation rates of $1 \mathrm{ML}$ (monolayer)/min onto substrates kept at room temperature. NCAFM images were acquired at room temperature using a commercial AFM (VT-AFM, Omicron Nanotechnology $\mathrm{GmbH} 65232$ Taunusstein, Germany) equipped with homebuilt electronics and an RHK controller (SPM 1000 plus PLL Pro II/R9, RHK Technology Inc., Troy, MI 48083, USA). Nanosensor cantilevers were used (PPP-NCL with resonance frequency $f_{0}=150 \mathrm{kHz}$, spring constant $k=30 \mathrm{~N} / \mathrm{m}$, quality factor $Q=40000)$ at oscillation amplitudes $A_{0}$ of $10 \mathrm{~nm}$. Experimental NC-AFM images were analyzed by using the WSxM software. ${ }^{43}$ All distances and directions were calibrated with respect to the ionic crystal surface from atomically resolved images of the substrate.

Classical Force Fields. To study self-assembled film structures and their dynamics, classical force fields were employed since the computational cost associated with DFT calculations of a large number of molecules of this size at the surface is too high. All classical calculations were performed using the LAMMPS code, ${ }^{44}$ where the interactions inside the $\mathrm{KCl}$ substrate were described using a Buckingham potential parametrized by Catlow et $\mathrm{al}^{45}$ and the inter- and intramolecular interactions of $\mathrm{CDB}$ and $\mathrm{TCB}$ molecules were described using the CHARMM force field. ${ }^{46}$ A particle-particle particle-mesh (P3M) solver was used to compute the longrange Coulomb interactions to an accuracy of $10^{-4}$. The force fields for the interaction of $\mathrm{CDB}$ and TCB molecules with the $\mathrm{KCl}$ (001) surface have been derived in our previous publications (see refs 42 and 25, respectively), where more details of the parametrization and evaluation of these force fields can be found.

Briefly, a training set of 240 configurations was generated using van der Waals corrected DFT methods, and a genetic algorithm (GA) was applied to fit parameters of a force field against that data set. A total population of 1024 elements were chosen for the GA scheme and evolved over 1000 generations, with $5 \%$ of the total populations being randomized in each generation to reduce artificial convergence. The DFT calculations of the data set were previously used to study the adsorption energy, geometry, and electronic structure of CDB and TCB molecules on $\mathrm{KCl}(001){ }^{22,25}$ These calculations were performed using the CP2K code, the PBE GGA density functional, and a mixed Gaussian and plane wave basis set, where we employed semiempirical long-range dispersion corrections $^{47}$ to describe vdW interactions in the system. Finally, the MOLOPT ${ }^{48}$ basis set was selected to minimize basis set superposition error, and a plane-wave cutoff of $400 \mathrm{Ry}$ was used. This produced a band gap of $5.4 \mathrm{eV}$, surface rumpling of $0.3 \AA$, and a lattice constant of $6.3 \AA$ for $\mathrm{KCl}(001)$, in agreement with experimental values. ${ }^{49} \mathrm{We}$ assessed the accuracy of the vdW corrections employed against higher accuracy second-order Møller-Plesset perturbation theory $(\mathrm{MP} 2)^{50,51}$ calculations of smaller molecular fragments. ${ }^{25}$

Using the GA scheme, the best fit to the $a b$ initio data was obtained for a Morse-type interatomic potential combined with point charges to represent the electrostatic interactions, where classical charges of \pm 1 were used for $\mathrm{KCl}$ and the charges on $\mathrm{TCB} / \mathrm{CDB}$ atoms were derived from Mulliken population analysis of the DFT results. The adsorption energies obtained from this force field compare within a $7 \%$ deviation with the 
data obtained by vdW-corrected DFT calculations, and the adsorption geometries are reproduced well.

The setup for all theoretical simulations consisted of a fourlayer slab of $\mathrm{KCl}(001)$ with the bottom layer fixed in its bulk position to prevent the system from drifting in the simulation box. The temperature was controlled by NVT thermostats (Nose-Hoover), which were applied to the surface and the molecules separately. Molecular dynamics simulations were run using a 1 fs time step and were equilibrated for 1 ps. A sample input script and force field files can be found in the Supporting Information.

Entropy Change Calculations. We have previously shown that entropy loss upon adsorption of CDB and TCB molecules on a clean terrace greatly contributes to the adsorption free energy and is comparable to the enthalpy contribution at high temperatures. $^{25}$ Further entropy loss can occur when the rotation of molecules on a surface terrace is constrained at step edges and in molecular structures. For large physisorbed molecules of the type considered here, changes in rotational entropy play a dominant role. A rough estimate of the rotational entropy of a single molecule can be obtained using a rigid rotor model. The rotational entropy of a molecule is estimated relative to a state where the molecule does not rotate and the barrier for rotation is assumed to be zero, meaning the molecule is free to rotate $360^{\circ}$ around its center of mass as given by

$$
\begin{aligned}
& T \cdot \bar{S}_{\text {rot }}^{\text {ads }}=k_{\mathrm{B}} T\left[\frac{1}{2}+\ln \left(z_{\text {rot }}^{\text {ads }}\right)\right] \\
& z_{\text {rot }}^{\text {ads }}=\frac{1}{\sqrt{\pi} \sigma}\left(\frac{8 \pi^{2} k_{\mathrm{B}} T}{h^{2}}\right)^{1 / 2}\left(I_{z}\right)^{1 / 2}
\end{aligned}
$$

The symmetry factor $\sigma$ denotes how many configurations are identical if the molecule is rotated by $360^{\circ}$ around the surface normal, and the moment of inertia is given by $I_{z}=\sum_{i} m_{i}\left(x_{i}^{2}+\right.$ $y_{i}^{2}$ ), where $x_{i}$ and $y_{i}$ are the molecule coordinates with the origin at its center of mass and the $z$ axis pointing along the surface normal.

A more accurate estimate of entropy changes can be attained using a method proposed by Smith et al., ${ }^{52}$ where the change in entropy is obtained via thermodynamic integration as given by

$$
\begin{aligned}
-T S(r)= & -T S\left(r_{0}\right)+\frac{1}{k T} \int_{r_{0}}^{r} \mathrm{~d} R\left[\left\langle E \frac{\partial E}{\partial R}\right\rangle_{R}\right. \\
& \left.-\langle E\rangle\left\langle\frac{\partial E}{\partial R}\right\rangle_{R}\right]
\end{aligned}
$$

where $E$ is the potential energy and $R$ is a defined reaction coordinate. In our case, the reaction coordinate was defined as the distance between a molecule and a step edge or a center of mass (COM) of another molecule. The average is taken over $50 \mathrm{~ns} \mathrm{MD}$ simulations, after a 1 ps equilibration time. Using shorter simulation times is not sufficient to sample the configuration space. The convergence of our results was checked by increasing the MD simulation time to $80 \mathrm{~ns}$ since with longer simulation times more of the high energy, low probability phase space will be sampled, which is critical for obtaining accurate entropy changes. Zero entropy was defined to be at large separation where the force between the molecule and the step or between two molecules goes to zero. The reaction coordinate $R$ was varied in $0.1 \AA$ intervals, and the center of mass was fixed at each $R$.

\section{RESULTS}

NC-AFM Imaging of CDB and TCB Molecules on $\mathrm{KCl}$ (001). The self-assembly of flexible CDB molecules was previously studied on the $\mathrm{KCl} \mathrm{(001)} \mathrm{surface} \mathrm{at} \mathrm{room}$ temperature, ${ }^{22}$ where they were shown to form large areas of defect-free, porous networks. The more rigid TCB molecules were synthesized, deposited onto $\mathrm{KCl}(001)$, and also imaged at room temperature using NC-AFM. The adsorption behavior of these two molecules is compared in order to gain insight into the effects of molecular flexibility on nucleation, growth, and morphology of film structures.

When CDB molecules are deposited onto a clean $\mathrm{KCl}$ (001) surface, step edges are decorated before molecular islands are formed. Figure la shows homogeneous bright rims, which run
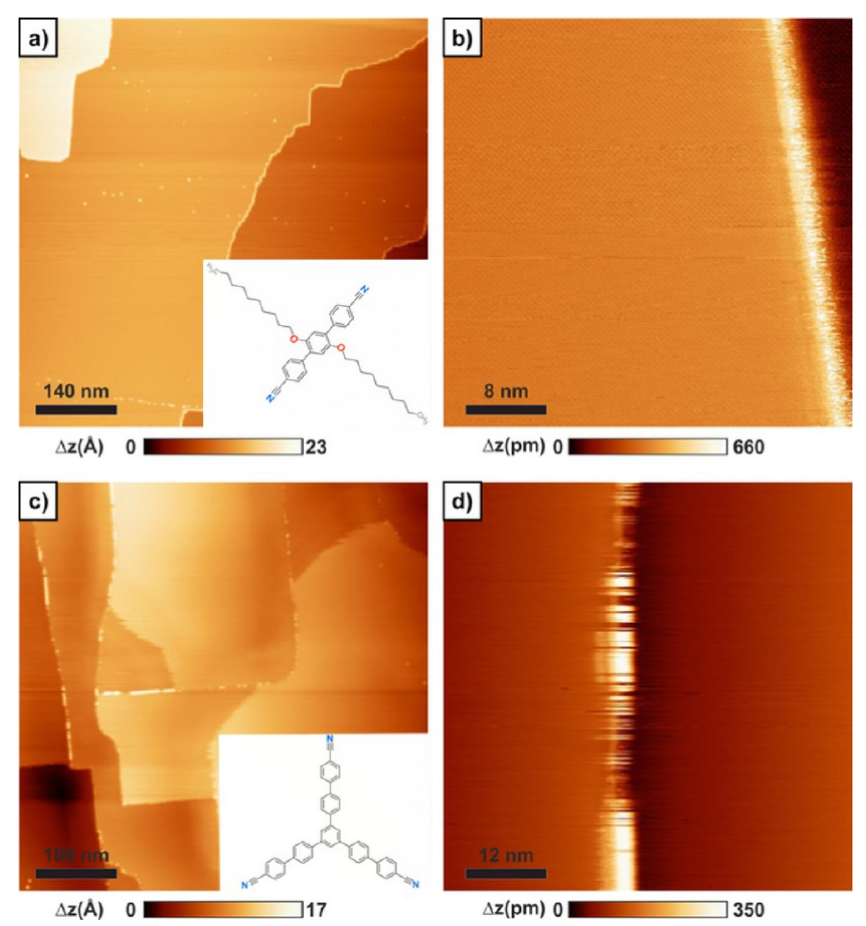

Figure 1. (a) Topography image where 0.05 monolayers (ML) of $\mathrm{CDB}$ molecules was deposited onto $\mathrm{KCl}$ (001). In the upper left corner there is a terrace, which is completely covered by $\mathrm{CDB}$ molecules, and all step edges are fully decorated. (b) A topography image of 0.05 ML of CDB molecules deposited onto $\mathrm{KCl}$ (001) showing a decorated step edge. (c) A topography image of TCB molecules deposited onto $\mathrm{KCl}$ (001), which shows mostly undecorated step edges and fuzzy stripes. These reproducible fuzzy stripes represent areas where molecules diffuse along crystallographically oriented steps. (d) A representative zoomed-in image over a fuzzy step edge area. A few of the bright objects are stably imaged; however, larger fuzzy stripes can be observed over several scans.

along all $\mathrm{KCl}$ steps at low molecule coverage. One terrace is completely covered by CDB molecules (upper left corner), and some bright clusters are observed on terraces. The latter are impurities on the surface due to sample preparation (ex situ cleaving and only in situ annealing). Individual molecules were not visible at room temperature, implying that they may be rapidly diffusing across the surface. Furthermore, imaging individual molecules at step edges was not possible (Figure 1b), most probably due to the mobility of the hydrocarbon chains. 


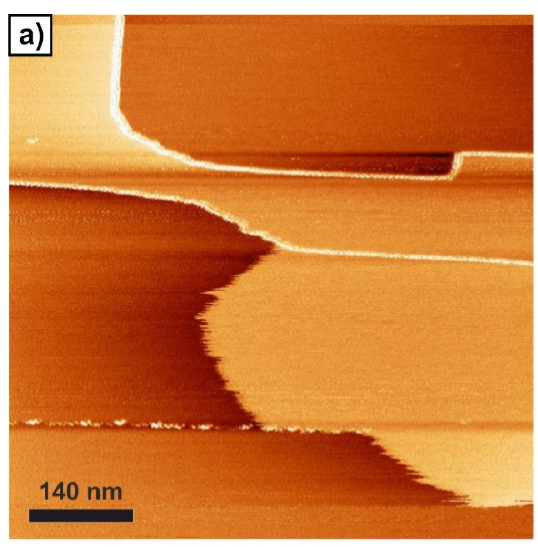

$\Delta \mathbf{z}(\mathbf{A})$

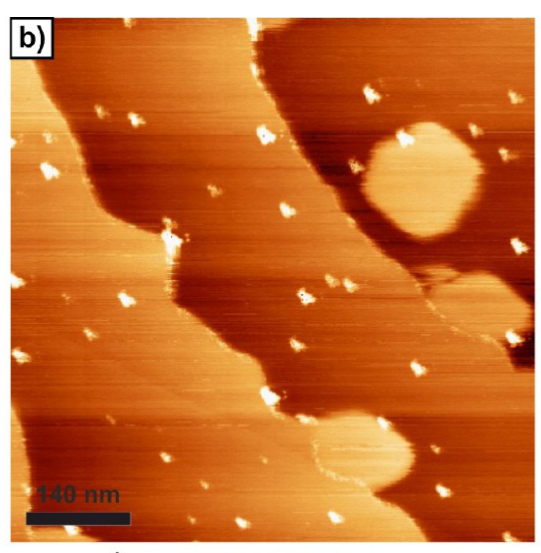

$\Delta \mathrm{z}(\mathbf{A}) 0$

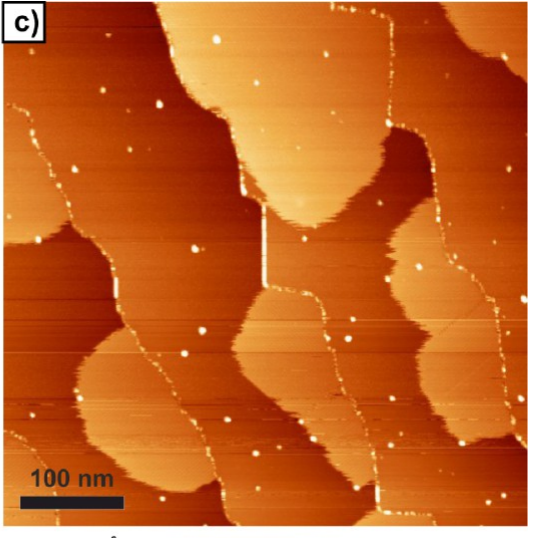

$\Delta z(\AA) 0 \square$

Figure 2. (a) $0.15 \mathrm{ML}$ of $\mathrm{CDB}$ deposited on $\mathrm{KCl}$ : Island borders are unstable, and molecular layers diffuse on the surface. All step edges are decorated. (b) $0.1 \mathrm{ML}$ and (c) $0.3 \mathrm{ML}$ coverage of TCB on $\mathrm{KCl}$ : typical drop-like islands growing on terraces and steps and few smaller islands observed to diffuse on the surface.
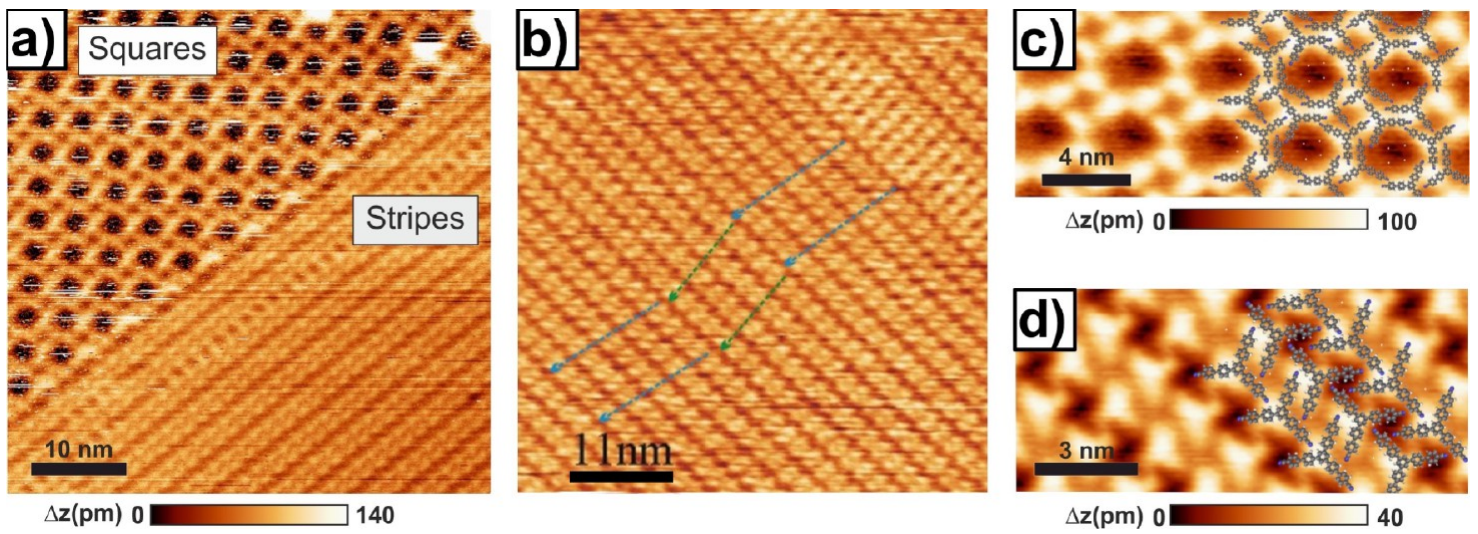

Figure 3. (a) Overview of both the porous structure and the line structure formed by TCB molecules on the $\mathrm{KCl}(001)$ surface at room temperature. (b) Large linear domain showing alternating orientations of the line structure. (c) A zoomed-in mesh average image of the square porous structure along with a proposed atomistic model. (d) A zoomed-in mesh average image of the denser line structure along with a proposed atomistic model.

In contrast, TCB molecules do not completely cover the substrate step edges (see Figure 1c and d), even at coverages as large as 0.6 ML. Two main features can be observed at step edges. The first is that the step edges aligned with the crystallographic direction of $\mathrm{KCl}(001)$ exhibit fuzzy stripes. This might be explained if TCB molecules are attracted to the step edge by the AFM tip. Second, we observe stable bright features at kink sites. These could be explained by either trapped TCB molecules or impurities.

These results provide evidence that $\mathrm{CDB}$ films readily nucleate at step edges, while TCB films do not and may nucleate on clean terraces instead. This should result in qualitatively different growth modes for the two molecules. $\mathrm{CDB}$ molecular films should grow from step edges on the $\mathrm{KCl}$ (001) surface, while self-assembled films composed of TCB molecules should also exhibit island growth on clean terraces. Indeed some island growth was observed at room temperature, as shown in Figure $2 \mathrm{~b}$.

At higher coverages, CDB forms very large molecular islands, which often appear to be single domains, as published previously. ${ }^{22}$ Even at low coverages no small molecular clusters could be observed, as displayed in Figure 1a, but instead molecules would form large islands, which often completely decorate a terrace. The large island size is an indication of fast diffusion with a large diffusion length, and large molecular islands were observed to diffuse as a single domain on the surface. The edges of an island appear fuzzy and are unstable under repeated scanning of the same area. For TCB at low coverage $(\sim 0.1 \mathrm{ML})$ small islands with drop like shapes can be observed to grow on terraces (Figure $2 b$ ). These small islands are mobile and diffuse over the surface under repeated scanning; however, once they reach a step edge they get immobilized. This is an indication of a different growth mode compared to $\mathrm{CDB}$, where small isolated islands were not observed on terraces. At higher coverages and smaller terraces ( $\sim 0.3 \mathrm{ML}$, Figure $2 \mathrm{c})$, TCB islands are more often observed at step edges, however, still with drop-like shapes and rarely decorating complete terraces, whereas CDB islands often span $>1 \mu \mathrm{m}^{2}$.

The two self-assembled structures formed by TCB molecules are shown in Figure $3 \mathrm{a}$ and can be termed as a line structure and a porous network structure. The line structure is the denser packed film at $2.5 \times 10^{-3} \mathrm{TCB} / \AA^{2}$ and repeats with a lattice of about $(17 \AA \times 31 \AA) \pm 2 \AA$. In comparison, the square structure has a density of $2.3 \times 10^{-3} \mathrm{TCB} / \AA^{2}$ and a lattice of 41 $\AA \times 41 \AA$. Both film structures can be observed to coexist on the surface and are stable at $300 \mathrm{~K}$, as no transition between one to the other can be observed over time. However, heating the system up to $370 \mathrm{~K}$ leads to the transition from the square structure to the denser line structure. Several features could also 
be observed within the line structure, such as line splitting (Figure $3 a$ ), varying orientations of the lines (Figure $3 b$ ), as well as different contrasts (Figure $3 b$ top right corner). These indicate the presence of several stable polymorphs of the line structure at room temperature.

These experimental results indicate that $\mathrm{CDB}$ molecules and TCB molecules follow different growth dynamics. CDB molecules spend more time at step edges, where nucleation may start, while TCB molecules do not. Therefore, CDB molecular films may grow from step edges on the $\mathrm{KCl}(001)$ surface, while self-assembled films composed of TCB molecules exhibit island growth on clean terraces as well as step edges.

In order to understand the TCB film structure and get some insight into its growth mechanisms and molecular dynamics, we used the following approach. Since the balance of interactions is crucial for our predictions, we first need to test our force fields in action. The information provided by experiment suggests fast diffusion and shows the difference in the character of the surface step decoration for the two molecules. We therefore first model the molecular diffusion, the interaction of $\mathrm{CDB}$ and TCB molecules with the surface steps, and the pairwise interaction between the TCB molecules. We seek qualitative agreement with experiment to gain confidence in the force fields and insight into the importance of entropic effects in these processes.

Molecular Diffusion. To study diffusion of CDB and TCB molecules, classical MD calculations were performed for $30 \mathrm{~ns}$ by placing a single molecule on the $\mathrm{KCl}(001)$ surface with the plane of the molecule parallel to the surface plane. From the molecular trajectories, the diffusion coefficients and diffusion barrier can be calculated using a standard mean-squareddisplacement approach (as outlined in refs 53 and 54). However, at a temperature of $300 \mathrm{~K}$ the TCB molecule was observed to diffuse very slowly, and the $30 \mathrm{~ns}$ simulation time was not enough to accurately calculate the diffusion coefficient. Diffusion coefficients calculated from the trajectories at elevated temperatures are given in Table 1 . The magnitude of the

Table 1. Diffusion Coefficients of TCB and CDB Molecules on a $\mathrm{KCl}(001)$ Surface as Calculated from MD Trajectories Using a Mean-Squared Displacement Approach

\begin{tabular}{ccc} 
& \multicolumn{2}{c}{ diffusion coefficients $/\left(\mathrm{cm}^{2} / \mathrm{s}\right)$} \\
\cline { 2 - 3 } temperature & TCB & CDB \\
$450 \mathrm{~K}$ & $1.13 \times 10^{-7}$ & $6.49 \times 10^{-7}$ \\
$500 \mathrm{~K}$ & $4.38 \times 10^{-7}$ & $2.56 \times 10^{-6}$ \\
$550 \mathrm{~K}$ & $1.33 \times 10^{-6}$ & $2.26 \times 10^{-5}$ \\
\hline
\end{tabular}

diffusion coefficients compares well with the coefficients found for similar systems. ${ }^{55}$ Diffusion barrier calculated from the gradient of a linear fit to an Arrhenius plot of the diffusion coefficients vs inverse temperature amounts to $0.52 \mathrm{eV}$ for TCB and $0.36 \mathrm{eV}$ for $\mathrm{CDB}$.

A more detailed understanding of the diffusion dynamics can be gained by further analysis of the COM trajectories of these two molecules and their configurations which are shown in Figure 4 for $30 \mathrm{~ns}$ simulations at $500 \mathrm{~K}$.

The COM of TCB sits preferably above a cation ( $\mathrm{K}$ ion) site. The molecule rotates in a plane parallel to the surface with the cyano groups preferentially pointing toward cations. The trajectory shows that the molecule's COM does not cross an anion site but instead prefers to move diagonally in between two anion sites. This means that at least two legs have to move
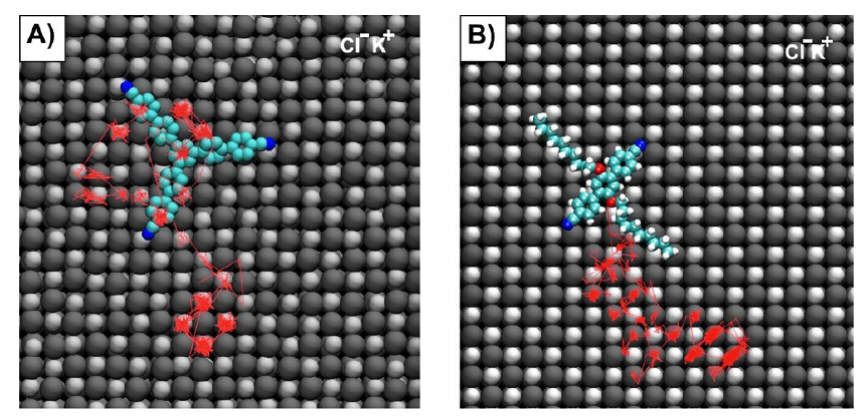

Figure 4. Diffusion of a TCB (A) and a CDB (B) molecule at $500 \mathrm{~K}$. The center of mass trajectory of a MD simulation is illustrated by the red trace. Note that for clarity in (B) the COM was computed of the central benzene ring body only since the arms display fast and erratic motion.

to neighboring cation sites in order to facilitate this translation. Due to the rigidity of the molecule and a strong interaction between the surface cations and the cyano groups, this motion has a large barrier, and the molecule spends a considerable amount of time with its COM above the same cation site, leading to slower diffusion overall. This seems to point toward an epitaxial model where the COM has well-defined adsorption sites on the $\mathrm{KCl}$ lattice. Clips of $\mathrm{MD}$ simulations illustrating the molecular motion can be found in the Supporting Information.

In contrast, $\mathrm{CDB}$ exhibits faster diffusion with a lower diffusion barrier. The diffusion mechanism also exhibits some major differences. First, CDB has many local minima close in energy, which can be thermally accessed, in contrast to TCB, which does not. Thus, the flexible hydrocarbon arms move easily over the surface, occasionally lifting up off the surface at $T=300 \mathrm{~K}$ and above. The central axis of the body of the molecule also has several different orientations with respect to the surface axes and can point along [110], [100], or [310] directions. The combination of many accessible local minima and the lower diffusion barrier results in overall more rapid diffusion. It is also worth noting that an epitaxial model of growth seems less likely to work in this case as the molecule displays a plethora of conformations on the surface, unlike the TCB molecule.

These results are in good agreement with experimental NCAFM images taken at room temperature, where single molecules cannot be resolved on clean $\mathrm{KCl}$ (001) terraces. Due to the large difference between the time scales relevant for molecular diffusion and those relevant to NC-AFM imaging, both the $\mathrm{CDB}$ and $\mathrm{TCB}$ molecule would undergo many rotations and translations during the time needed to collect a single experimental image at room temperature. These mobile individual molecules are therefore not expected to be imaged, unless they are stabilized by a feature such as a step edge, kink, or other trapping site.

Interaction of TCB and CDB Molecules with Step Edges and Kinks. Modeling the interaction of TCB and CDB with step edges and kinks provides a very sensitive test for the balance of molecule-surface interactions because they characterize the difference provided by the specific interaction with a relatively small number of step and kink atoms with respect to the strong interaction with the terrace mainly governed by the vdW forces. The experimental evidence suggests that at room temperature $\mathrm{CDB}$ molecules are more strongly bound to steps than $\mathrm{TCB}$, which we will now examine 

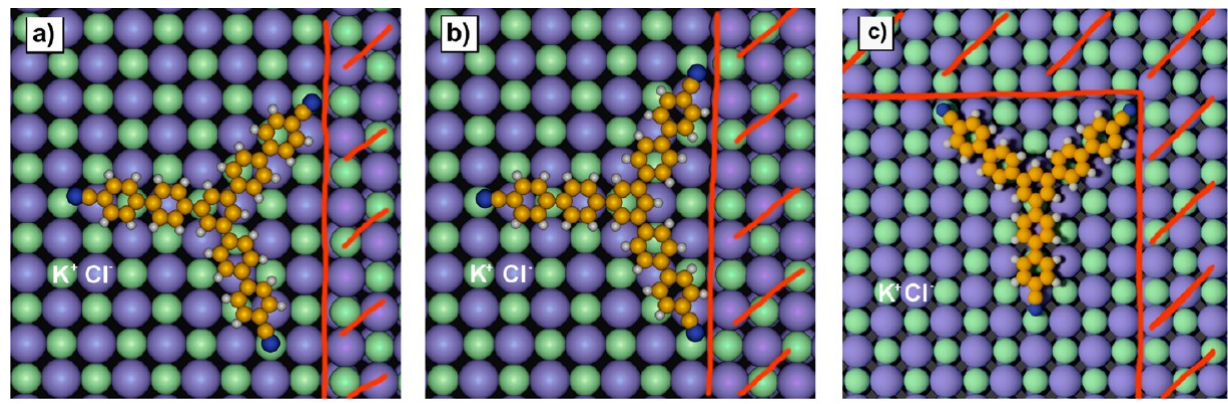

Figure 5. Two possible adsorption geometries for a TCB molecule at a monatomic step edge are shown along with the adsorption geometry at a kink site. Both configurations are energetically favorable in comparison to adsorption on a clean terrace. (a) The TCB molecule adsorbs with one $\mathrm{CN}$ group positioned near the step edge cation site. (b) The TCB molecule distorts to adsorb with two CN groups positioned near step edge cations. (c) The TCB molecule adsorbs with one $\mathrm{CN}$ group in the kink site and a second $\mathrm{CN}$ group positioned near the step edge at a cation site.

to find out why. These results can be also related to the initial stages of film nucleation observed experimentally.

TCB Molecules at Step Edges and at Kinks. To investigate the interaction of TCB molecules with the $\langle 100\rangle$ monatomic step edge on the $\mathrm{KCl}$ (001) surface, the lower terrace was represented using three atomic layers of $\mathrm{KCl}$. A $50 \mathrm{~ns} \mathrm{MD}$ simulation of 100 TCB molecules, corresponding to about 0.5 ML coverage, on a slab containing a step edge was first performed at $500 \mathrm{~K}$ to increase molecular mobility. At this temperature, TCB molecules rotate rapidly and have sufficient energy to overcome the diffusion barriers necessary to reach the step edge. They were observed most often adapting a configuration with two legs attached to the step with the angle between legs stretching so that $\mathrm{CN}$ functional groups terminating each of the legs can interact with cation sites at the upper step edge. On average about $75 \%$ of the step is covered with molecules, where $60 \%$ of these molecules adapt a geometry with two legs interacting with the step edge, while the rest are only able to interact using one functional group.

Using this information we performed energy minimization calculations to study the adsorption geometry of individual TCB molecules at a step edge. They confirmed that there are three possible configurations. In the first configuration, only one leg of the molecule is attached to the step at a cation site (see Figure 5a). This geometry is very similar to the previously calculated $^{25}$ adsorption geometry for TCB on a clean $\mathrm{KCl}$ (001) terrace. The interaction energy of the molecule with the step amounts to $0.15 \mathrm{eV}$, giving a total adsorption energy of 4.7 $\mathrm{eV}$.

In the second configuration, two legs interact with $\mathrm{K}^{+}$sites on the step edge. However, the angle between these two legs is forced to stretch and distort in order to accomplish this (see Figure $5 b$ ). Despite the distortion of the molecule, the adsorption energy in this geometry was calculated to be 4.8 $\mathrm{eV}$. This again highlights the strong interaction between the $\mathrm{CN}$ groups and the step cation sites. A second molecule gains about $0.1 \mathrm{eV}$ from adsorbing on the step edge next to an already adsorbed molecule in a configuration with one leg attached to the step and another pointing toward the COM of the already adsorbed molecule.

Finally, in the third configuration the angle between the molecule's legs decreases instead of stretching in order to attach two legs to two $\mathrm{K}^{+}$sites at the step edge. This reduces the adsorption energy to $4.5 \mathrm{eV}$. This value is slightly smaller than the $4.55 \mathrm{eV}$ adsorption energy calculated for an isolated TCB molecule on the $\mathrm{KCl}$ (001) terrace, and thus this configuration will be unstable over longer periods of time. Indeed this configuration is very short-lived in $\mathrm{MD}$ simulations, and the molecule quickly adopts one of the other two more stable configurations on the step edge. Overall these results are consistent with our molecular dynamics observations, where the energetically favorable configuration with two legs attached to the step edge is most commonly observed.

The calculations of TCB adsorption at kink sites show that in the most energetically favorable configuration one leg of the molecule is pointing into the kink and attaching to the two cations, which corresponds to the molecule interacting with a total of three cation sites, as illustrated in Figure 5c. Notably the most stable configuration at a kink site is when the angle between the two attached legs is being compressed rather than stretched, in contrast to the results at a step edge. This is to maximize the interaction between the three cations of the kink and the cyano group pointing into the kink. The adsorption energy of the optimized configuration was calculated to be 5.1 eV. A TCB molecule adsorbing next to a molecule adsorbed at a kink site has an adsorption energy of about $4.8 \mathrm{eV}$ with only one leg attached to the step edge and one pointing toward the COM of the molecule in the kink site.

CDB Molecules at Step Edges and Kinks. Similarly, long time-scale simulations of $\mathrm{CDB}$ molecules on the $\mathrm{KCl}(001)$ surface with the [100] step edge at $500 \mathrm{~K}$ have been used to explore possible adsorption configurations accompanied by energy minimization calculations.

The lowest energy configuration of the CDB molecule at the step edge shown in Figure 6a has the adsorption energy of 4.0 $\mathrm{eV}$ compared to $3.1 \mathrm{eV}$ on a clean terrace. This increase in the adsorption energy can be attributed to two main factors: (i) that the $\mathrm{CN}$ group can simultaneously interact with two cation sites on the surface rather than just one and (ii) the hydrocarbon chain lies along the step edge which further increases the adsorption energy via the vdW interaction with the upper step atoms. This result is consistent with the experimental images that show decorated step edges at low coverages of $\mathrm{CDB}$ molecules at room temperature (see Figure 2a). After one CDB molecule has been stabilized at the step edge, it becomes increasingly favorable for additional molecules to adsorb there. The lowest energy configuration of a second captured molecule adsorbing adjacent to the first one along the step edge was calculated to be $4.2 \mathrm{eV}$.

At kink and corner sites, the CDB adsorption energy increases further as at these sites, and the $\mathrm{CN}$ group can interact with a total of three cation sites. The second hydrocarbon chain can now lie along a step edge as well. One such configuration is shown in Figure $6 \mathrm{~b}$. Calculations of a 


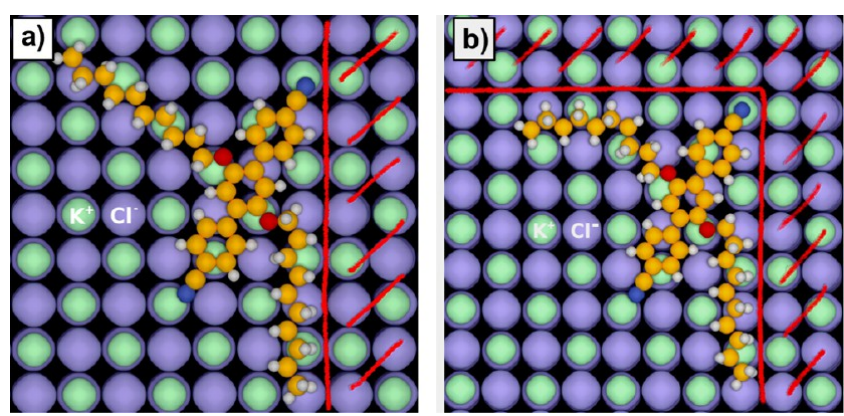

Figure 6. (a) Optimized adsorption geometry for a single $\mathrm{CDB}$ molecule at a step edge on the $\mathrm{KCl}(001)$ surface. The $\mathrm{CN}$ anchoring group simultaneously interacts with two surface cations, while the hydrocarbon chain adsorbs along the step edge itself. (b) The optimized adsorption geometry for a single CDB molecule at a kink feature on the $\mathrm{KCl}$ (001) surface. The $\mathrm{CN}$ anchoring group simultaneously interacts with three surface cations, while the two hydrocarbon chains lie along the step edges. These interactions contribute to greatly increase the adsorption energy of the molecule.

$\mathrm{CDB}$ molecule adsorbed at a kink on $\mathrm{KCl}$ (001) give the adsorption energy of $4.4 \mathrm{eV}$. Thus, molecules captured at kinks are more stable than at step edges. These molecules were predicted to increase the adsorption energy of adjacent molecules along the step edge to $4.2 \mathrm{eV}$ and ones on the clean terrace to $3.3 \mathrm{eV}$ as well.

Comparing the two molecules, the conformational freedom of the more flexible $\mathrm{CDB}$ molecule allows it to adapt to maximize its interactions with the step edge or a kink site. In contrast, the more rigid TCB molecule has very little conformational freedom and is penalized when trying to adapt its geometry to fit cation adsorption sites.

Entropy Changes Due to Adsorption at Steps and Kinks. Since both molecules were observed to rotate rapidly on the surface at temperatures above $300 \mathrm{~K}$, but lose that rotational freedom when adhered to a step edge, rotational entropy loss can contribute significantly to their adsorption free energy at steps. Using a rigid rotor model we estimate that single TCB molecule would lose $\Delta S_{\text {rot }}=0.14 \mathrm{eV}$ and a single $\mathrm{CDB}$ molecule in the configuration with stretched out carbonyl chains $\Delta S_{\text {rot }}=0.15 \mathrm{eV}$ of rotational entropy and $0.14 \mathrm{eV}$ with the arms aligned along the aromatic body. However, these values must be regarded with caution as treating the flexible $\mathrm{CDB}$ molecule as a rigid body is a crude model since the hydrocarbon chains move rapidly in our MD simulations.

Thermodynamic integration calculations can provide a better estimate. ${ }^{56}$ This method relies on averaging over all configurations of the molecule in $\mathrm{MD}$ simulations at a set distance from the step edge, instead of predefining a fixed trajectory. The average change in entropy for TCB calculated in this way as a function of the distance of the COM of the molecule from the step at $300 \mathrm{~K}$ is shown in Figure 7. Upon adsorption at a step edge, the entropy of the molecule is reduced by about $0.2 \mathrm{eV}$. Initially the entropy decreases almost linearly as the molecule approaches the step edge and adheres with one leg. In this geometry the molecule retains some rotational freedom, but as the molecule gets closer to the step edge, two legs attach leading to a further drop in entropy. In that geometry the molecule does not rotate or translate and is essentially frozen. In the MD simulations of a single molecule at a step edge at $300 \mathrm{~K}$ it was observed that, in order to diffuse along the step edge, the molecule has to detach one leg from

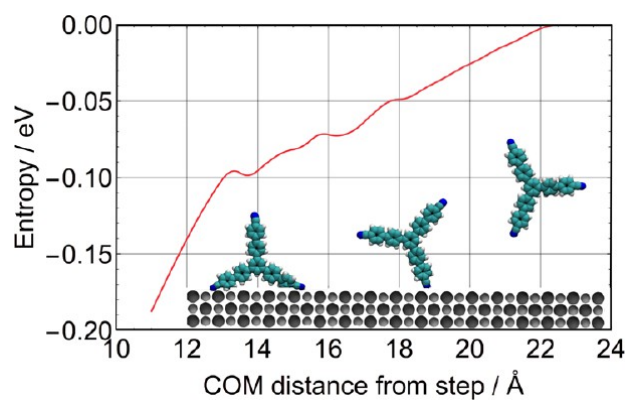

Figure 7. Change in entropy for a single TCB molecule as a function of the center of mass distance to the step edge at $300 \mathrm{~K}$. At $23 \AA$ the interaction between the molecule and the step edge is negligible, and at this separation the entropy was defined to be zero. Initially only one leg of the molecule attaches to the step edge, and a rapid decrease in entropy can be observed as two legs adhere to the step edge.

the step edge. Furthermore, in none of our simulations was the molecule observed to climb to the upper terrace of the step edge, indicating the presence of a Schwöbel barrier. While in energy minimization calculations the TCB molecule would gain $0.25 \mathrm{eV}$ by adsorbing on a step edge, this value is reduced due to entropic effects at higher temperatures. Thus, the adsorption energy is significantly lowered, which may explain that only partially covered step edges are observed by NC-AFM (see Figure 1d).

The change in entropy for CDB at $300 \mathrm{~K}$ shown in Figure 8 is almost three times larger for the flexible $\mathrm{CDB}$ molecule than

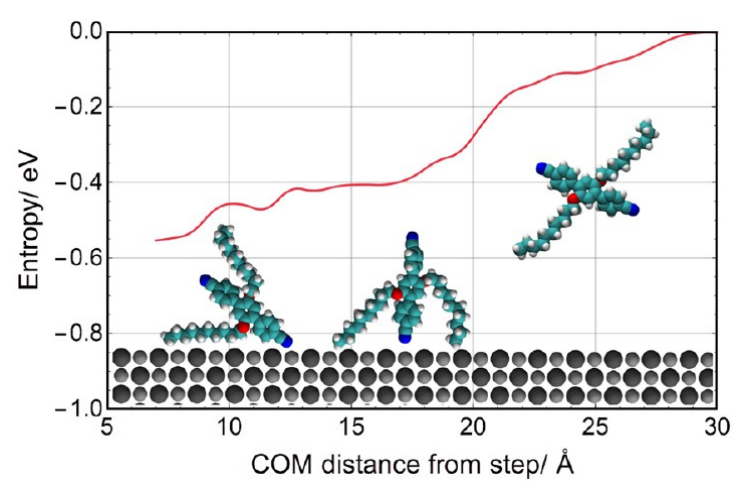

Figure 8. Change in entropy for a single CDB molecule as a function of the center of mass distance to a monatomic step edge at $300 \mathrm{~K}$. The initial decrease in entropy can be attributed to one arm of the CDB molecule adhering to the step edge. As the molecule gets even closer to the step, a cyano group will attach at a cation site of the step edge, leading to a further drop in entropy.

for the rigid TCB molecule. Upon adhesion to a step edge the $\mathrm{CDB}$ molecule loses $0.5 \mathrm{eV}$ of entropy. Initially only the very end of a carbonyl chain will attach to the step edge, but as the molecule gets closer, that chain starts adsorbing parallel to the step edge. The largest drop in entropy can be observed around $20 \AA$, where one carbonyl chain becomes completely attached along the step edge. Thus, the main contribution to the change in entropy comes from one of the very flexible carbonyl chains adsorbing along a step edge. However, despite the larger change in entropy, the free energy of adsorption on a step edge vs terrace is still larger for CDB than for TCB. Thus, CDB molecules favor to be adsorbed at step edges, in accordance to our NC-AFM data. 
Table 2. Overview of Thermodynamic Properties for TCB and CDB Molecules on KCl (001) ${ }^{a}$

\begin{tabular}{cccccc} 
& $\Delta H_{\text {ads }}^{\text {terrace }}$ & $-T \Delta S_{\text {ads }}^{\text {terrace }}$ & $\Delta F_{\text {ads }}^{\text {terace }}$ & \multicolumn{1}{c}{$\Delta H_{\text {ads }}^{\text {step }}$} & $-T \Delta S_{\text {ads }}^{\text {step }}$ \\
TCB & $-4.55 \mathrm{eV}$ & $0.5 \mathrm{eV}$ & $-4.05 \mathrm{eV}$ & $-4.8 \mathrm{eV}$ & $0.2 \mathrm{eV}$ \\
$\mathrm{CDB}$ & $-3.1 \mathrm{eV}$ & $1.0 \mathrm{eV}$ & $-2.1 \mathrm{eV}$ & $-4.0 \mathrm{eV}$
\end{tabular}

${ }^{a} \Delta H_{\text {ads }}^{\mathrm{X}}$ gives the adsorption enthalpy on a terrace or step edge, and $\Delta S_{\text {ads }}^{\mathrm{X}}$ gives the change in entropy for adsorption at $300 \mathrm{~K}$. The free energy $\Delta F_{\text {ads }}^{\mathrm{X}}$ can be calculated from $\Delta F_{\mathrm{ads}}^{\mathrm{X}}=\Delta H_{\mathrm{ads}}^{\mathrm{X}}-T \Delta S_{\mathrm{ads}}^{\mathrm{X}}$.

A summary of the values of enthalpy, entropy change, and free energy for adsorption of CDB and TCB is given in Table 2. The free energy is calculated as the difference between the enthalpy (adsorption energy at $0 \mathrm{~K}$ ) and the entropy change at $300 \mathrm{~K}$. While for TCB the free energy gain of adsorbing on a step edge is only $0.55 \mathrm{eV}$, the gain for $\mathrm{CDB}$ is significantly larger at $1.4 \mathrm{eV}$.

We also note that for a rigid TCB molecule the estimate using the rigid rotor model is very close to the value obtained from thermodynamic integration for the change in entropy of adhesion on a step edge. However, the rigid rotor model fails for the more flexible CDB molecule which displays a more complex step adhesion mechanism, where the molecule adapts its geometry to maximize its interaction with the step edge. Furthermore, translational motion of the molecule cannot be ignored since $\mathrm{CDB}$ has a higher diffusion coefficient and a lower diffusion barrier. Thus, only considering rotational entropy and treating the molecule as a rigid body results in an underestimation of the free energy change upon step adhesion.

Film Structure. The results presented above demonstrate that the force fields employed in this work provide a realistic qualitative picture of the interactions of individual molecules with terraces, steps, and kinks of the $\mathrm{KCl}$ (001) surface. We can now try to provide models of monolayers and compare them with the experimental NC-AFM images. In the earlier work we showed that $\mathrm{CDB}$ forms a square porous organic network with only two possible domain orientations on the $\mathrm{KCl}$ (001) surface. $^{22}$ The formation of this network is governed by the cyano groups $(\mathrm{CN})$, which dominated the molecule-substrate interaction by adsorbing on cationic sites of the substrate, and by the molecule-molecule interaction between the cyanophenyl groups and the hydrocarbon chains of neighboring molecules. In comparison, TCB self-assembles into two different structures, a linear and a porous network structure (see Figure 3a).

The balance between molecule-molecule (MM) and molecule-surface (MS) interactions determines if and how molecules self-assemble. Conventionally, a shallow potential energy surface (POT) allows the molecules to adjust their adsorption geometry to maximize the interactions with other molecules. However, if $\mathrm{MM}$ interactions are too strong, the molecules will form molecular crystals instead of $2 \mathrm{D}$ films. To get an insight into the local structures formed by TCB molecules we performed MD simulations of 100 TCB molecules on a $\mathrm{KCl}(001)$ surface (coverage of about 0.5 $\mathrm{ML})$ at 300,400 , and $500 \mathrm{~K}$ for $50 \mathrm{~ns}$. While most TCB molecules often change their orientation and coordination within $50 \mathrm{~ns}$, some small clusters were found to persist for longer periods of time (>10 ns). Within these small, structurally stable clusters TCB molecules could be seen forming short, between two and five molecule, chains, where one leg of a molecule points toward the central benzene ring of a neighboring molecule, as the cyano group forms hydrogen bonds with three hydrogens (Figure 9a). However, the
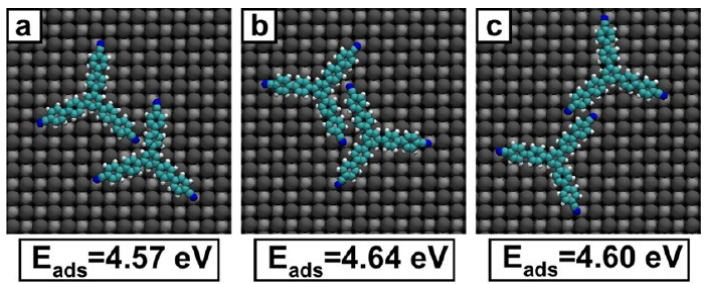

Figure 9. Three stable dimer configurations as observed in $\mathrm{MD}$ simulations. Energy minimization calculations were performed to find the energetically lowest geometries. The adsorption energy of a single TCB molecule in the respective configuration is given below each image.

molecules are distorted away from their lowest adsorption geometry in order to increase the MS interaction, leading to a small energy penalty of $0.1 \mathrm{eV}$ in the $\mathrm{MS}$ interaction. Additionally, in most of these clusters the dominant feature observed was the formation of TCB dimers, where a leg of one TCB molecule aligns with a leg of a neighboring molecule. The most stable configuration was found to be where two legs of the TCB molecules align side by side (Figure 9b). Since both molecules adapt the lowest energy adsorption geometry as found for an isolated molecule, there is no energy penalty associated with forming these dimers. The interaction energy between two TCB molecules in such a dimer configuration was calculated to be $0.2 \mathrm{eV}$ using energy minimization calculations. In the configuration shown in Figure $9 c$ the molecules also adapt the optimal adsorption geometry while interacting favorably through hydrogen bonds and dipole-dipole interactions. These structures are proposed to be the basic building blocks for both observed film structures and have also been observed in experimental studies of TCB on $\mathrm{Si}: \mathrm{B}(111) .^{57}$ However, these calculations do not include entropy change. The dimer formation significantly immobilizes TCB molecules, and it is interesting to compare the entropy change at room temperature upon formation of a TCB dimer with respect to isolated molecules on the surface.

Applying the same methodology as for step adhesion, the entropy loss of the dimer formation was calculated. The distance between the two molecules was fixed at each step, but the molecular motion was not frozen to allow both molecules to sample the entire configuration space. Each individual MD simulation was performed with a 1 fs time step over $50 \mathrm{~ns}$. Figure 10 illustrates the change in entropy as a function of the distance between COM of the two molecules. The equilibrium distance between the COM of the molecules as obtained from energy minimization calculations is indicated by the vertical black line. Forming a dimer of two TCB molecules induces an entropy reduction of about $0.1 \mathrm{eV}$. This can be compared with the interaction enthalpy of $0.2 \mathrm{eV}$ obtained using energy minimization calculations. Hence the TCB dimers are expected to form transiently and be stable for a considerable amount of time, as has been observed in our MD simulations. 


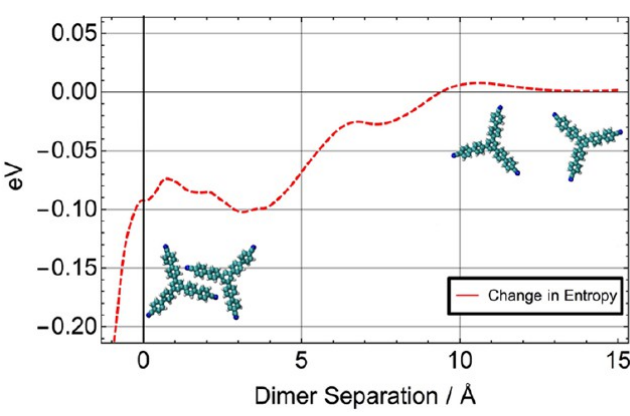

Figure 10. Plot illustrating the change in entropy as a function of molecule-molecule distance. Zero on the $x$ axis corresponds to the equilibrium dimer separation as obtained in energy minimization calculations.

The mobility of dimers signifies that direct simulation of the film growth process is unfeasible. However, the local structures observed in the MD simulations suggest clear motifs for constructing prototype periodic infinite structures for total energy calculations and geometry optimizations. The proposed structures are shown in Figure 11. The lowest adsorption
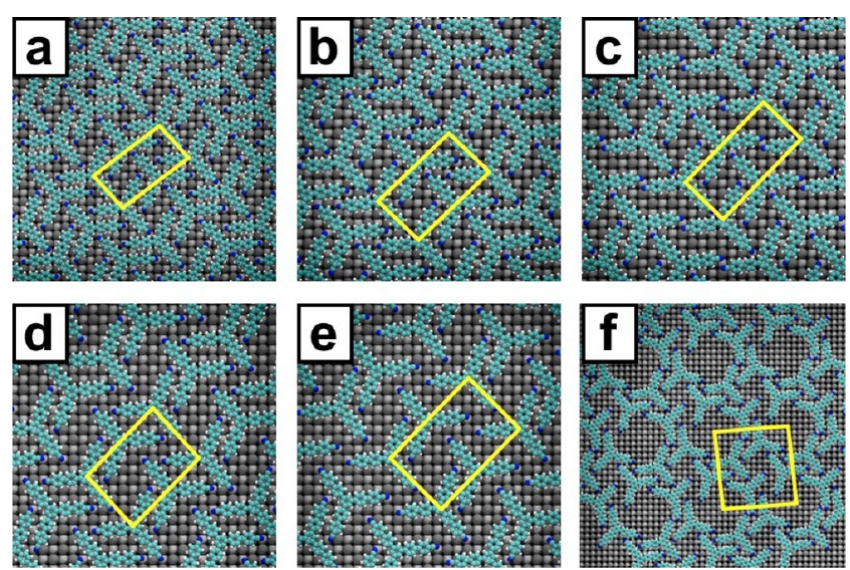

Figure 11. Proposed self-assembled structures for TCB molecules. The respective unit cell vectors are (a) $31.4 \AA \times 17.8 \AA$ with $\alpha=82^{\circ}$, (b) $31 \AA \times 17.8 \AA$ with $\alpha=90^{\circ}$, (c) $35.5 \AA \times 17.8 \AA$ with $\alpha=90^{\circ}$, (d) $31 \AA \times 22.2 \AA$ with $\alpha=90^{\circ}$, (e) $35.5 \AA \times 22.2 \AA$ with $\alpha=90^{\circ}$, and (f) $40.9 \AA \times 40.9 \AA$ with $\alpha=90^{\circ}$.

energy was found for configuration (b) at $4.75 \mathrm{eV}$ followed by configuration (c) at $4.73 \mathrm{eV}$ per molecule. This is consistent with the experimentally observed structures (Figure 3a), where the configuration (b) reproduces well the experimentally measured film unit cell and the observed line splitting (Figure $1 \mathrm{a}, \mathrm{b})$ is due to the energetically close structure (c). The configuration (f) was found to be the lowest energy geometry for the porous network film with an adsorption energy of 4.65 $\mathrm{eV} / \mathrm{molecule}$. Despite smaller adsorption energy than for the linear structure, large domains of the porous network are observed experimentally at room temperature. This can arise from entropic contributions at nonzero temperatures not accounted for in enthalpy calculations above. As the molecules in the porous network are not as densely packed and constrained as in the line structure, the entropic cost of adsorbing in a film should be lower.

To probe the film stability, we performed molecular dynamics simulations starting from the lowest enthalpy structures using an (NVT) ensemble at 300 and $400 \mathrm{~K}$. The proposed line structure was found to be stable at both temperatures throughout a $40 \mathrm{~ns}$ simulation. At the domain boundary there was no diffusion of molecules observed at 300 $\mathrm{K}$. However, at $400 \mathrm{~K}$ some molecules at the boundary rotated and broke out from the film. Since the molecules at the edge of the island are less confined by others, they can more easily overcome the barrier required to break away.

The molecules in the center of the porous structure were observed to be stable at $300 \mathrm{~K}$, but the molecules at the boundary were less confined and occasionally broke out of the porous geometry. At $400 \mathrm{~K}$ this effect became more pronounced until the structure broke apart entirely after about $20 \mathrm{~ns}$, resulting in a disordered structure. These results are consistent with the experimental NC-AFM observations, where upon annealing the system at $120{ }^{\circ} \mathrm{C}$ only the higher density line structure could be observed. The formation of the dimers was found to be the critical feature in both film structures, which has also been reported in experimental observations of TCB molecules on $\mathrm{Si}: \mathrm{B}(111),{ }^{57}$ where the lower density network was found to be the energetically favorable one, as it maximized the number of dimer interactions. We also note that the proposed TCB layer structures are similar to the dense and porous structures proposed for other tripod molecules on different substrates, confirming their universal nature. ${ }^{36-41}$

\section{DISCUSSION AND CONCLUSIONS}

Despite progress in recent years, nucleation and growth mechanisms of monolayers and ordered films of organic molecules at insulating surfaces are still poorly understood. It is often assumed that step edges serve as nucleation sites, where molecules get trapped and film growth may start. This study sheds a new light on the possible dynamics of this process and the resulting structure.

In order for a well-defined monolayer to grow, molecules need to be able to diffuse across the surface to find stable nucleation sites, from where film growth may start. Previous calculations and experimental data agree that individual $\mathrm{CDB}$ and TCB molecules diffuse rapidly across the $\mathrm{KCl}$ (001) surface. Mobile $\mathrm{CDB}$ molecules are quickly trapped at step edges and kink sites on the $\mathrm{KCl}$ (001) surface. At these sites, the adsorption energy of the molecule is greatly increased due to its ability to adapt. The entropy loss due to reduced degrees of freedom is compensated by the conformational flexibility of the molecule, which allows it to maximize its interaction with steps and kinks, where ultimately film growth may start.

In contrast, the rigid TCB molecule displays different dynamics. Since it is unable to adapt its geometry to step edges and kinks, it can only interact with the step edge through $\mathrm{CN}$ functional groups. However, even then some energy is lost due to the molecular distortions required to position both $\mathrm{CN}$ groups near cation sites on the step edge. With weak step edge interaction energy, the entropy loss upon adhesion on step edges becomes significant and destabilizes the molecule. The NC-AFM images taken at room temperature confirm that TCB molecules can nucleate on terraces and thus display a different film-growth mechanism to CDB molecules. These mechanisms can be used to understand differences in images taken at low coverage, where CDB molecules are clearly visible at step edges and kinks, while TCB molecules are noticeably absent.

Molecules bonded to insulators by the non-site-specific molecule-surface vdW interaction often display complex or no epitaxial growth. However, the addition of anchoring groups 
that bind to specific surface sites has been reported to lead to epitaxial growth, and attempts to apply the concept of epitaxy to organic thin films have been made. ${ }^{26,58,59}$ However, organic molecules can have complex geometries and low plane group symmetry, thus introducing greater complexity. In particular the balance between intermolecular interactions and moleculesurface interactions dictates whether a molecular film will grow commensurate to the substrate lattice or in a more complex structure. TCB molecules have three anchoring cyano groups, which interact strongly with the $\mathrm{KCl}$ surface, leading to the molecules assembling on specific sites of the $\mathrm{KCl}(001)$ lattice. The intermolecular interactions are not strong enough to perturb the molecule significantly from its minimum adsorption geometry leading to "point-on-point" commensurism. To summarize, our results show that molecular flexibility and entropic contributions to adsorption play an important role in not only the morphology of self-assembled films but also the growth process itself. Tuning the conformational flexibility and functionality of molecules are powerful parameters that can be used to design molecules for self-assembly.

\section{ASSOCIATED CONTENT}

\section{S Supporting Information}

The Supporting Information is available free of charge on the ACS Publications website at DOI: 10.1021/acs.jpcc.6b12738.

Video of a MD simulation of TCB step adhesion on $\mathrm{KCl}$ (001) (MPG)

Video of TCB diffusion on a $\mathrm{KCl}$ (001) terrace (MPG) Videos of $\mathrm{CDB}$ diffusion on a $\mathrm{KCl}(001)$ terrace (MPG) LAMMPS input scripts and force field parameters (PDF)

\section{AUTHOR INFORMATION}

\section{Corresponding Authors}

*E-mail: julian.gaberle.10@ucl.ac.uk.

*E-mail: david.gao.10@ucl.ac.uk.

*E-mail: christian.loppacher@im2np.fr. Phone: + +1.831 .233 .3329 .

ORCID $\odot$

Julian Gaberle: 0000-0002-4414-0588

Notes

The authors declare no competing financial interest.

\section{ACKNOWLEDGMENTS}

The authors acknowledge financial support from the ANR NANOKAN program (ANR-11-BS100004). JG is supported by EPSRC and JAIST, Japan. JG, DZG, and ALS acknowledge the use of the ARCHER high-performance computing facilities via our membership to the U.K.s HPC Materials Chemistry Consortium, which is funded by EPSRC (grant EP/L000202). DZG acknowledges the use of the Serenity computing cluster and support services provided by Nanolayers Research Computing for the completion of this work. The authors would like to thank F. Federici Canova for his help in parametrizing the force field for TCB and A. Schwarz and M. Jenei for useful discussions.

\section{REFERENCES}

(1) Mason, A.; Mukhopadhyay, S. C.; Jayasundera, K. P. Sensing Technology: Current Status and Future Trends I; Springer: New York City, USA, 2015.
(2) Chaki, N. K.; Vijayamohanan, K. Self-Assembled Monolayers as a Tunable Platform for Biosensor Applications. Biosens. Bioelectron. 2002, 17, 1-12.

(3) Maboudian, R; Ashurst, W. R; Carraro, C. Self-assembled Monolayers as Anti-Friction Coatings for MEMS: Characteristics and Recent Developments. Sens. Actuators, A 2000, 82, 219-223.

(4) Besenbacher, F.; Lauritsen, J. V.; Wendt, S. STM Studies of Model Catalysts. Nano Today 2007, 2, 30-39.

(5) Joachim, C.; Gimezewski, J. K.; Aviram, A. Electronics Using Hybrid-Molecular and Mono-Molecular Devices. Nature 2000, 408, 541-548.

(6) Heath, J. R. Molecular Electronics. Annu. Rev. Mater. Res. 2009, $39,1-23$.

(7) Song, H.; Reed, M. A.; Lee, T. Single Molecule Electronic Devices. Adv. Mater. 2011, 23, 1583-1608.

(8) deWild, M.; Berner, S.; Suzuki, H.; Yanagi, H.; Schlettwein, D.; Ivan, S.; Baratoff, A.; Guntherodt, H.-J.; Jung, T. A. A Novel Route To Molecular Self-Assembly: Self-Intermixed Monolayer Phases. ChemPhysChem 2002, 3, 881-885.

(9) Bobisch, C.; Wagner, T.; Bannani, A.; Moller, R. Ordered Binary Monolayer Composed of Two Organic Molecules: CopperPhthalocyanine and 3,4,9,10-Perylene-Tetra-Carboxylic-Dianhydride on $\mathrm{Cu}(111)$. J. Chem. Phys. 2003, 119, 9804-9808.

(10) Grill, L.; Dyer, M.; Lafferentz, L.; Persson, M.; Peters, M. V.; Hecht, S. Nano-Architectures by Covalent Assembly of Molecular Building Blocks. Nat. Nanotechnol. 2007, 2, 687-691.

(11) Abel, M.; Clair, S.; Ourdjini, O.; Mossoyan, M.; Porte, L. Single Layer of Polymeric Fe-Phtalocyanine: An Organometallic Sheet on Metal and Thin Insulating Film. J. Am. Chem. Soc. 2011, 133, 12031205.

(12) Ourdjini, O.; Pawlak, R.; Abel, M.; Clair, S.; Chen, L.; Bergeon, N.; Sassi, M.; Oison, V.; Debierre, J. M.; Coratger, R.; et al. SubstrateMediated Ordering and Defect Analysis of a Surface Covalent Organic Framework. Phys. Rev. B: Condens. Matter Mater. Phys. 2011, 84, 125421.

(13) Payamyar, P.; Kaja, K.; Ruiz-Vargas, C.; Stemmer, A.; Murray, D. J.; Johnson, C. J.; King, B. T.; Schiffmann, F.; VandeVondele, J.; Renn, A.; et al. Synthesis of a Covalent Monolayer Sheet by Photochemical Anthracene Dimerization at the Air/Water Interface and its Mechanical Characterization by AFM Indentation. Adv. Mater. 2014, 26, 2052-2058.

(14) Zheng, Z.; Opilik, L.; Schiffmann, F.; Liu, W.; Bergamini, G.; Ceroni, P.; Lee, L.-T.; Schutz, A.; Sakamoto, J.; Zenobi, R.; et al. Synthesis of Two-Dimensional Analogues of Copolymers by Site-toSite Transmetalation of Organometallic Monolayer Sheets. J. Am. Chem. Soc. 2014, 136, 6103-6110.

(15) Baris, B.; Jeannoutot, J.; Luzet, V.; Palmino, F.; Rochefort, A.; Cherioux, F. Noncovalent Bicomponent Self-Assemblies on a Silicon Surface. ACS Nano 2012, 6, 6905-6911.

(16) Hinaut, A.; Pujol, A.; Chaumeton, F.; Martrou, D.; Gourdon, A.; Gauthier, S. An NC-AFM and KPFM Study of the Adsorption of a Triphenylene Derivate on $\mathrm{KBr}(001)$. Beilstein J. Nanotechnol. 2012, 3, 221-229.

(17) Hauke, C. M.; Bechstein, R.; Kittelmann, M.; Storz, C.; Kilbinger, A. F. M.; Rahe, P.; Kühnle, A. Controlling Molecular SelfAssembly on an Insulating Surface by Rationally Designing an Efficient Anchor Functionality That Maintains Structural Flexibility. ACS Nano 2013, 7, 5491-5498.

(18) Pawlak, R.; Nony, L.; Bocquet, F.; Oison, V.; Sassi, M.; Debierre, J. M.; Loppacher, C.; Porte, L. Supramolecular Assemblies of 1,4-Benzene Diboronic Acid on $\mathrm{KCl}(001)$. J. Phys. Chem. C 2010, 114, 9290-9295.

(19) Rahe, P.; Nimmrich, M.; Kühnle, A. Templating: Substrate Templating Upon Self-Assembly of Hydrogen-Bonded Molecular Networks on an Insulating Surface. Small 2012, 8, 2968-2968.

(20) Loppacher, C.; Zerweck, U.; Eng, L. M.; Gemming, S.; Seifert, G.; Olbrich, C.; Morawetz, K.; Schreiber, M. Adsorption of PTCDA on a Partially $\mathrm{KBr}$ Covered $\mathrm{Ag}(111)$ Substrate. Nanotechnology 2006, $17,1568-1573$. 
(21) Hentschke, R.; Schürmann, B. L.; Rabe, J. P. Molecular Dynamics Simulations of Ordered Alkane Chains Physisorbed on Graphite. J. Chem. Phys. 1992, 96, 6213-6221.

(22) Amrous, A.; Bocquet, F.; Nony, L.; Loppacher, C.; Palmino, F.; Cherioux, F.; Gao, D. Z.; Canova, F. F.; Watkins, M. B.; Shluger, A. L.; et al. Molecular Design and Control Over the Morphology of SelfAssembled Films on Ionic Substrates. Adv. Mater. Interfaces 2014, 1, 1400414.

(23) Schwarz, A.; Gao, D. Z.; Lämmle, K.; Grenz, J.; Watkins, M. B.; Shluger, A. L.; Wiesendanger, R. Determining Adsorption Geometry, Bonding, and Translational Pathways of a Metal-Organic Complex on an Oxide Surface: Co-Salen on $\mathrm{NiO}(001)$. J. Phys. Chem. C 2013, 117, $1105-1112$.

(24) Hoff, B.; Gingras, M.; Peresutti, R.; Henry, C. R.; Foster, A. S.; Barth, C. Mechanisms of the Adsorption and Self-Assembly of Molecules with Polarized Functional Groups on Insulating Surfaces. J. Phys. Chem. C 2014, 118, 14569-14578.

(25) Gaberle, J.; Gao, D. Z.; Canova, F. F.; Watkins, M. B.; Shluger, A. L. Calculating the Entropy Loss on Adsorption of Organic Molecules at Insulating Surfaces. J. Phys. Chem. C 2016, 120, 39133921.

(26) Rahe, P.; Kittelmann, M.; Neff, J. L.; Nimmrich, M.; Reichling, M.; Maass, P.; Kühnle, A. Tuning Molecular Self-Assembly on Bulk Insulator Surfaces by Anchoring of the Organic Building Blocks. Adv. Mater. 2013, 25, 3948-3956.

(27) Chatterjee, A.; Vlachos, D. G. An Overview of Spatial Microscopic and Accelerated Kinetic Monte Carlo Methods. J. Comput.-Aided Mater. Des. 2007, 14, 253-308.

(28) Abbasi-Pérez, D.; Manuel Recio, J.; Kantorovich, L. The Role of Isomerization in the Kinetics of Self-Assembly: P-terphenyl-mdicarbonitrile on the $\operatorname{Ag}(111)$ Surface. Phys. Chem. Chem. Phys. 2015, 17, 11182-11192.

(29) Silly, F.; Weber, U. K.; Shaw, A. Q.; Burlakov, V. M.; Castell, M. R.; Briggs, G. A. D.; Pettifor, D. G. Deriving Molecular Bonding from a Macromolecular Self-Assembly using Kinetic Monte Carlo Simulations. Phys. Rev. B: Condens. Matter Mater. Phys. 2008, 77, 201408.

(30) Ortmann, F.; Schmidt, W. G.; Bechstedt, F. Attracted by LongRange Electron Correlation: Adenine on Graphite. Phys. Rev. Lett. 2005, 95, 186101.

(31) Fajín, J. L. C.; Teixeira, F.; Gomes, J. R. B.; Cordeiro, M. N. D. S. Effect of van der Waals Interactions in the DFT Description of SelfAssembled Monolayers of Thiols on Gold. Theor. Chem. Acc. 2015, 134, 67.

(32) Kemball, C. Adv. Catal.; Academic Press: New York City, USA, 1950; Vol. II.

(33) Campbell, C. T.; Sellers, J. R. V. Enthalpies and Entropies of Adsorption on Well-Defined Oxide Surfaces: Experimental Measurements. Chem. Rev. 2013, 113, 4106-4135.

(34) Waldmann, T.; Klein, J.; Hoster, H. E.; Behm, R. J. Stabilization of Large Adsorbates by Rotational Entropy: A Time-Resolved Variable-Temperature STM Study. ChemPhysChem 2013, 14, 162169.

(35) Guo, T.; Yang, J.; Zeng, L.; Wang, H.; Tong, Q.; Li, X. Does There Exist an Intrinsic Relationship Between the Flexibility and SelfAssembly of Pepfactants. Mol. Simul. 2014, 40, 423-430.

(36) Szabelski, P.; Rzysko, W.; Nieckarz, D. Directing the SelfAssembly of Tripod Molecules on Solid Surfaces: A Monte Carlo Simulation Approach. J. Phys. Chem. C 2016, 120, 13139-13147.

(37) Ciesielski, A.; Szabelski, P. J.; Rzysko, W.; Cadeddu, A.; Cook, T. R.; Stang, P. J.; Samorí, P. Concentration-Dependent Supramolecular Engineering of Hydrogen-Bonded Nanostructures at Surfaces: Predicting Self-Assembly in 2D. J. Am. Chem. Soc. 2013, 135, 6942-6950.

(38) Bellec, A.; Arrigoni, C.; Schull, G.; Douillard, L.; FioriniDebuisschert, C.; Mathevet, F.; Kreher, D.; Attias, A. J.; Charra, F. Solution-Growth Kinetics and Thermodynamics of Nanoporous SelfAssembled Molecular Monolayers. J. Chem. Phys. 2011, 134, 124702.

(39) Gutzler, R.; Sirtl, T.; Dienstmaier, J. F.; Mahata, K.; Heckl, W. M.; Schmittel, M.; Lackinger, M. Reversible Phase Transitions in Self-
Assembled Monolayers at the Liquid-Solid Interface: TemperatureControlled Opening and Closing of Nanopores. J. Am. Chem. Soc. 2010, 132, 5084-5090.

(40) Tahara, K.; Furukawa, S.; Uji-I, H.; Uchino, T.; Ichikawa, T.; Zhang, J.; Mamdouh, W.; Sonoda, M.; De Schryver, F. C.; De Feyter, S.; et al. Two-Dimensional Porous Molecular Networks of Dehydrobenzo 12 Annulene Derivatives via Alkyl Chain Interdigitation. J. Am. Chem. Soc. 2006, 128, 16613-16625.

(41) Feyter, S. D.; Mali, K. S. Principles of Molecular Assemblies Leading to Molecular Nanostructures. Philos. Trans. R. Soc., A 2013, 371, 20120304.

(42) Gao, D. Z.; Federici Canova, F.; Watkins, M. B.; Shluger, A. L. Efficient Parametrization of Complex Molecule-Surface Force Fields. J. Comput. Chem. 2015, 36, 1187-1195.

(43) Horcas, I.; Fernandez, R.; Gomez-Rodriguez, J. M.; Colchero, J.; Gomez-Herrero, J.; Baro, A. M. WSXM: A Software for Scanning Probe Microscopy and a Tool for Nanotechnology. Rev. Sci. Instrum. 2007, 78, 013705.

(44) Plimpton, S. Fast Parallel Algorithms for Short-Range Molecular Dynamics. J. Comput. Phys. 1995, 117, 1-19.

(45) Catlow, C. R. A.; Diller, K. M.; Norgett, M. J. Interionic Potentials for Alkali Halides. J. Phys. C: Solid State Phys. 1977, 10, $1395-1412$

(46) Brooks, B.; Bruccoleri, R.; Olafson, D.; States, D.; Swaminathan, S.; Karplus, M. CHARMM: A Program for Macromolecular Energy, Minimization and Dynamics Calculations. J. Comput. Chem. 1983, 4, 187-217.

(47) Grimme, S. Semiempirical GGA-type Density Functional Constructed With a Long-Range Dispersion Correction. J. Comput. Chem. 2006, 27, 1787-1799.

(48) VandeVondele, J.; Hutter, J. Gaussian Basis Sets for Accurate Calculations on Molecular Systems in Gas and Condensed Phases. J. Chem. Phys. 2007, 127, 114105.

(49) Vogt, J.; Weiss, H. The Structure of $\mathrm{NaCl}\left(\begin{array}{lll}1 & 0 & 0\end{array}\right)$ and $\mathrm{KCl}\left(\begin{array}{ll}1 & 0\end{array}\right.$ 0) Single Crystal Surfaces: a Tensor Low Energy Electron Diffraction Analysis. Surf. Sci. 2001, 491, 155-168.

(50) Del Ben, M.; Hutter, J.; VandeVondele, J. Second-Order MøllerPlesset Perturbation Theory in the Condensed Phase: An Efficient and Massively Parallel Gaussian and Plane Waves Approach. J. Chem. Theory Comput. 2012, 8, 4177-4188.

(51) Del Ben, M.; Hutter, J.; VandeVondele, J. Forces and Stress in Second Order Møller-Plesset Perturbation Theory For Condensed Phase Systems Within the Resolution-of-Identity Gaussian and Plane Waves Approach. J. Chem. Phys. 2015, 143, 102803.

(52) Smith, D. E.; Zhang, L.; Haymet, A. D. J. Entropy of Association of Methane in Water: A New Molecular Dynamics Computer Simulation. J. Am. Chem. Soc. 1992, 114, 5875-5876.

(53) Ma, M.; Tocci, G.; Michaelides, A.; Aeppli, G. Fast Diffusion of Water Nanodroplets on Graphene. Nat. Mater. 2016, 15, 66-71.

(54) Gomer, R. Diffusion of Adsorbates on Metal Surfaces. Rep. Prog. Phys. 1990, 53, 917-1002.

(55) Weckesser, J.; Barth, J. V.; Kern, K. Direct Observation of Surface Diffusion of Large Organic Molecules at Metal Surfaces: PVBA on $\mathrm{Pd}(110)$. J. Chem. Phys. 1999, 110, 5351-5354.

(56) Trzesniak, D.; Kunz, A.-P. E.; van Gunsteren, W. F. A Comparison of Methods to Compute the Potential of Mean Force. ChemPhysChem 2007, 8, 162-169.

(57) Copie, G.; Cleri, F.; Makoudi, Y.; Krzeminski, C.; Berthe, M.; Cherioux, F.; Palmino, F.; Grandidier, B. Surface-Induced Optimal Packing of Two-Dimensional Molecular Networks. Phys. Rev. Lett. 2015, 114, 066101.

(58) Hooks, D. E.; Fritz, T.; Ward, M. D. Epitaxy and Molecular Organization on Solid Substrates. Adv. Mater. 2001, 13, 227-241.

(59) Ward, M. D. Soft Crystals in Flatland: Unraveling Epitaxial Growth. ACS Nano 2016, 10, 6424-6428. 